\title{
An XPS Study of Bromine in Methanol Etching and Hydrogen Peroxide Passivation Treatments for Cadmium Zinc Telluride Radiation Detectors
}

\author{
S.Babar, P.J.Sellin, J.F.Watts, M.A.Baker \\ Faculty of Engineering and Physical Sciences, University of Surrey, \\ Guildford, Surrey, GU2 7XH, UK
}

Corresponding author: M.A.Baker e-mail: M.Baker@surrey.ac.uk

\begin{abstract}
The performance of single crystal CdZnTe radiation detectors is dependent on both the bulk and the surface properties of the material. After single crystal fabrication and mechanical polishing, modification of the surface to remove damage and reduce the surface leakage current is generally achieved through chemical etching followed by a passivation treatment. In this work, CdZnTe single crystals have been chemically etched using a bromine in methanol (BM) treatment. The BM concentrations employed were 0.2 and $2.0 \mathrm{v} / \mathrm{v} \%$ and exposure times varied between 5 and $120 \mathrm{~s}$. Angle resolved XPS and sputter depth profiling has been employed to characterize the surfaces for the different exposure conditions. A Te rich surface layer was formed for all exposures and the layer thickness was found to be independent of exposure time. The enriched Te layer thickness was accurately determined by calibrating the sputter rate against a CdTe layer of known thickness. For BM concentrations of $0.2 \mathrm{v} / \mathrm{v} \%$ and $2 \mathrm{v} / \mathrm{v} \%$, the Te layer thickness was determined to be $1.3 \pm 0.2$ and 1.8 $\pm 0.2 \mathrm{~nm}$ respectively. The BM etched surfaces have subsequently been passivated in a $30 \mathrm{wt} . \% \mathrm{H}_{2} \mathrm{O}_{2}$ solution employing exposure time of $15 \mathrm{~s}$. The oxide layer thickness has been calculated using two standard XPS methodologies, based on the Beer-Lambert expression. The $\mathrm{TeO}_{2}$ thickness calculated from ARXPS data are slightly higher than the thickness obtained by simplified Beer-Lambert expression. For BM exposure 30-120 s followed by passivation treatment of $30 \mathrm{wt} . \% \mathrm{H}_{2} \mathrm{O}_{2}$ solution employing exposure time $15 \mathrm{~s}$, ARXPS method gave $\mathrm{TeO}_{2}$ thickness $1.25 \pm 0.12 \mathrm{~nm}$ and for simplified Beer-Lambert expression the value was in the range of $1.0 \pm 0.07 \mathrm{~nm}$.
\end{abstract}

Keywords: Cadmium Zinc Telluride, CZT, XPS, passivation, oxide thickness. 


\section{Introduction}

X-ray and gamma rays detectors fabricated from single crystal cadmium zinc telluride (CZT) are able to operate at room temperature with low leakage currents [1]. The energy resolution of such detectors are enhanced by the wide band gap $(1.5-2.2 \mathrm{eV})$ and the higher atomic number $(\mathrm{Z})$ density of CZT [2],[3]. There is a correlation between the performance (charge generation, charge transport and intrinsic electric field) and the surface properties of CZT, hence optimising the specific surface properties are important to the detector performance [4]-[6]. After slicing and dicing of the crystal from its ingot, it is mechanically polished to reduce surface roughness. This is followed by chemical polishing which both further improves the roughness and removes microstructural damage resulting from the mechanical polishing process. The surface composition after chemical treatment depends on the chemical solution employed. A chemical treatment using an acidic solution produces a Te-rich surface, while an alkaline solution etch gives a Cd-rich surface [7]. Most commonly, bromine in methanol (BM) of varying concentrations is used. Özsan et al found that the $\mathrm{Cd} / \mathrm{Te}$ ratio at the surface was invariant after a 2 minute exposure in 0.2 and $1.0 \% \mathrm{BM}$ solution, but the graded Te layer increased in thickness as the BM concentration was increased [8]. However, the enriched Te layer thickness was not quantified. Aging of the BM solution increases its acidity, resulting in an enrichment of elemental Te at the surface. Rouse at al found that once the Te layer reaches a thickness of approximately $2 \mathrm{~nm}$, it is independent of chemical etching duration and the acidity of BM solution [9]. The authors used XPS depth profiling to determine the thickness, but do not describe how the $\mathrm{Ar}^{+}$ ion sputter rate employed was determined. Accurate quantification of the Te layer thickness is one of the primary aims of the work undertaken in this study.

Bensalah et al have shown that variation in BM exposure affects the surface roughness and leakage current [10]. The Te enrichment changes the stoichiometry of the surface and due to smaller band gap $(\sim 0.3 \mathrm{eV})$, increases the leakage current, deteriorating the radiation detector performance. To minimize the leakage current, the Te rich surface is passivated to develop a thin insulating oxide layer. Özsan et al showed that passivation of BM etched surfaces with 30 wt. $\% \mathrm{H}_{2} \mathrm{O}_{2}$ produces an oxide thickness which is dependent on BM concentration up to a BM concentration of $2 \%$ [8]. The oxide thickness dependence on exposure time of passivating agent was only measured for a $0.2 \%$, BM concentration, but was found to be similar, for all exposure times [8]. Considering a number of authors' work, the thickness of the $\mathrm{H}_{2} \mathrm{O}_{2}$ generated oxide is in the range of $1.6-4.5 \mathrm{~nm}$, depending on 
the BM concentration and oxide thickness calculation method employed $[8,11,[12]$.

In this paper, CZT single crystals have been chemically etched using BM concentrations of 0.2 and $2.0 \mathrm{v} / \mathrm{v} \%$ for varying times $(5-120 \mathrm{~s})$ and changes in surface composition determined by XPS depth profiling. An $\mathrm{Ar}^{+}$ion sputter rate of CZT has been evaluated by $\mathrm{Ar}^{+}$ion sputtering a CdTe thin film of known thickness. This has enabled an accurate value of the Te layer thickness to be determined for the different BM exposures. These BM etched surfaces were further passivated using a 30 wt. $\% \mathrm{H}_{2} \mathrm{O}_{2}$ solution and the oxide thickness determined from XPS data using two different methods: (i) the standard Beer-Lambert expression employing a single photoelectron take-off angle; (ii) a Beer-Lambert based methodology using a range of photoelectron take-off angles. A comparison is made of the oxide thickness determined by these different analytical methods.

\section{Experimental procedure}

\subsection{Sample preparation}

Cadmium Zinc Telluride (CZT) single crystal samples grown using the modified vertical Bridgman method were obtained from Yinnel Tech Inc., USA. The samples had dimensions of $8 \times 8 \times 5 \mathrm{~mm}^{3}$. The two samples had different bulk compositions, determined by quantitative energy dispersive X-ray (EDX) analysis to be $\mathrm{Cd}_{0.8} \mathrm{Zn}_{0.2} \mathrm{Te}$ and $\mathrm{Cd}_{0.9} \mathrm{Zn}_{0.1} \mathrm{Te}$. (The quantified EDX data was recorded using a Hitachi S3200 SEM employing an Oxford Instruments X-ray detector and the spectra quantified using the INCA software). Each sample was mechanically polished, with the final polish being performed using a $0.05 \mu \mathrm{m}$ alumina suspension in ethanediol. The samples were then dipped in freshly prepared BM solutions with concentrations of either 0.2 or $2.0 \mathrm{v} / \mathrm{v} \%$ for different time durations $(5,15,30,60$ or 120s). After BM exposure, the samples were rinsed with iso-propanol and kept in methanol to avoid oxidation. The samples were removed from the methanol immediately prior to being placed in the ultra-high vacuum of the XPS instrument. After each step during the chemical treatment, the samples were blow-dried with nitrogen gas. The solution $\mathrm{pH}$ was determined using universal $\mathrm{pH}$ indicator paper. The accuracy of the $\mathrm{pH}$ indicator was estimated to be the measured $\mathrm{pH} \pm 1$. For the passivation treatment the procedure above was repeated followed by exposure in $30 \mathrm{wt} . \%$ of $\mathrm{H}_{2} \mathrm{O}_{2}$ for 15s. After each BM or passivation treatment the CZT surface was re-polished using the alumina suspension ready for the next exposure.

To determine the $\mathrm{Ar}^{+}$sputter rate of CZT; a CdTe layer was deposited by metal organic chemical vapour deposition (MOCVD) onto a Si (100) wafer at the Centre for Solar Energy Research, Glyndŵr University, UK. Details of the process can be found in [13]. The nominal CdTe layer thickness, measured by in-situ laser interference reflectometry was $120 \mathrm{~nm}$. Using ex-situ mechanical 
profilometry, the thickness of the area to be $\mathrm{Ar}^{+}$sputtered was determined to be $135 \mathrm{~nm}$.

\subsection{XPS Analysis}

A Thermo Scientific Thetaprobe XPS instrument employing a monochromatic $\mathrm{Al} \mathrm{K}_{\alpha} \mathrm{X}$-ray source with a photon energy of $1486.68 \mathrm{eV}$ was used in this work. The diameter of the X-ray beam spot was $800 \mu \mathrm{m}$. survey spectra were recorded at a pass energy of $300 \mathrm{eV}$ and high resolution core level spectra recorded at pass energy of $20 \mathrm{eV}$. Quantification of the XPS data was performed after a Shirley background subtraction using the Thermo Scientific Avantage software which employs instrument modified Wagner sensitivity factors. Depth profiling was undertaken using an EX05 $\mathrm{Ar}^{+}$ ion gun operating at $3 \mathrm{kV}$ and current density of $11.1 \mu \mathrm{A} / \mathrm{cm}^{2}(1 \mu \mathrm{A}$ induced beam current, rastered over a $3 \times 3 \mathrm{~mm}^{2}$ area). Spectra were charge referenced to the $\mathrm{C} 1 \mathrm{~s}$ peak at $285.0 \mathrm{eV}$.

\section{Results and Discussion}

\subsection{CdTe sputter rate}

A depth profile of the MOCVD CdTe thin film is shown in Figure 1. The Cd:Te ratio exhibits a stoichiometry of 1.0:1.0 and the sputter rate for CdTe at $\mathrm{Ar}^{+}$ion bombardment at an energy of $3 \mathrm{kV}$ and beam current density of $11.1 \mu \mathrm{A} / \mathrm{cm}^{2}$ was determined to be $0.15 \mathrm{~nm} / \mathrm{s}$. This etch rate was then used to calibrate the depth scale for all the XPS depth profiles of BM etched CZT surfaces.

\subsection{CZT surface composition after BM etching}

The changes in surface composition from exposing CZT in $0.2 \mathrm{v} / \mathrm{v} \% \mathrm{BM}$ for times of 5, 15, 30, 60 and $120 \mathrm{~s}$ is shown by the XPS profiles given in Figure 2. For all exposure times, Te is clearly enriched at the surface with a progressive decrease in the Te concentration until the bulk concentration of 50 at.\% is attained. For all of the exposure times, there is little difference in surface composition and the Te rich layer has a thickness of $1.3 \pm 0.2 \mathrm{~nm}$. At the surface, the binding energy of the Te $3 \mathrm{~d}_{5 / 2}$ peak was found to be $572.9 \pm 0.1 \mathrm{eV}$ and this drops to a value of $572.5 \mathrm{eV}$ in the bulk, consistent with Te in a CZT matrix [10]. The bulk concentrations of $\mathrm{Cd}$ and $\mathrm{Zn}$ were found on average to be 37 and 13 at.\% respectively. It can be seen that Te enrichment occurs at the expense of both $\mathrm{Cd}$ and $\mathrm{Zn}$ and the XPS determined average surface concentrations for the different exposure times of Te, $\mathrm{Cd}$ and $\mathrm{Zn}$ are 66, 28 and 6 at.\% respectively (except for the 5s exposure, where the Te enrichment is slightly lower and the surface concentrations of $\mathrm{Te}, \mathrm{Cd}$ and $\mathrm{Zn}$ are 60,30 and 10 at.\% respectively).

The XPS profiles for CZT exposed to $2.0 \mathrm{v} / \mathrm{v} \%$ BM for times of 5, 15, 30, 60 and $120 \mathrm{~s}$ are presented in Figure 3. The profiles again show Te to be enriched at the surface in a similar manner for 
all of the exposure times with a progressive decrease in the Te concentration until the bulk concentration $(\mathrm{Te}=50$ at. $\%$ ) is reached. The Te enrichment for the $2.0 \mathrm{v} / \mathrm{v} \% \mathrm{BM}$ exposure is the same as for the $0.2 \mathrm{v} / \mathrm{v} \% \mathrm{BM}$ exposure, with the XPS results giving an average Te concentration for the different exposure times of 66 at.\% at the surface. The Te enrichment layer thickness has increased to $1.8 \pm 0.2 \mathrm{~nm}$ and is again invariant with exposure time except for the $5 \mathrm{~s}$ exposure duration thickness.

It is clear from these results that there is no difference in outer surface composition for either the two different BM concentrations or for different exposure times. These results are consistent both with the work of Rouse et al where no substantial differences in $[\mathrm{Cd}+\mathrm{Zn}] / \mathrm{Te}$ ratio were found for exposures of 1,3 and 9 min exposures in a $5 \%$ BM solution [8] and with Bensouici et al's [14] plot of $\mathrm{Te} /(\mathrm{Cd}+\mathrm{Zn})$ ratio for different BM concentrations of $1-4 \%$ which show very similar values for all $\mathrm{BM}$ concentrations [14][13]. However, the enriched Te layer thickness was found to increase slightly as the BM concentration was raised from 0.2 to $2.0 \%$, similar to the results of Özsan et al [8] . In our study, the pH of the 0.2 and $2.0 \%$ BM solutions is approximately 3 in both cases. The absence of a significant change in $\mathrm{pH}$ probably explains why there is not a large difference in the Te enrichment region observed for the different BM concentrations, and the relatively small increase in the enriched Te layer thickness may be caused by the $2 \mathrm{v} / \mathrm{v} \% \mathrm{BM}$ concentration having a slightly lower $\mathrm{pH}$ than the $0.2 \mathrm{v} / \mathrm{v} \% \mathrm{BM}$ solution. The invariance of the Te enrichment with exposure time may be explained by the thickness of the Te enriched surface layer being very small $(<2 \mathrm{~nm})$; thus the rate of reaction is so rapid that even very short exposure times do not influence the $\mathrm{Cd}$ and $\mathrm{Zn}$ etching process.

\subsection{Passivation treatment}

The effect of the passivation treatment (15 seconds exposure in $30 \mathrm{wt} . \% \mathrm{H}_{2} \mathrm{O}_{2}$ ) on the 0.2 and $2 \mathrm{v} / \mathrm{v}$ $\% \mathrm{BM}$ exposed surfaces can be seen from the changes in the Te $3 \mathrm{~d}_{5 / 2}$ peak presented in Figures 4 and 5. In addition to the elemental $\mathrm{Te} 3 \mathrm{~d}_{5 / 2}$ component at $572.8 \mathrm{eV}$, a peak at $576.3 \mathrm{eV}$ corresponding to $\mathrm{TeO}_{2}$ is observed. For the $0.2 \mathrm{v} / \mathrm{v} \% \mathrm{BM}$ treated surface (Figure 4), following passivation, the ratio of Te oxide peak to Te elemental peak $\left(\mathrm{Te}_{\mathrm{ox}} / \mathrm{Te}_{\mathrm{el}}\right)$ increases with $\mathrm{BM}$ exposure time up to $120 \mathrm{~s}$ of exposure, whilst for the $2.0 \mathrm{v} / \mathrm{v} \% \mathrm{BM}$ there is no difference in peak intensity ratio beyond an exposure time of $30 \mathrm{~s}$. This indicates that the oxide grown is slightly thicker for longer BM exposure times. XPS depth profiles plotted using the $\mathrm{Te}_{\mathrm{ox}}$ and $\mathrm{Te}_{\mathrm{el}}$ peaks for the $0.2 \mathrm{v} / \mathrm{v} \%$ and $2 \mathrm{v} / \mathrm{v} \% \mathrm{BM}$ exposed samples after passivation are given in Figures 6 and 7. All of the passivated surfaces show very similar behaviour, with the $\mathrm{Te}_{\mathrm{ox}}$ peak intensity being stronger than the $\mathrm{Te}_{\mathrm{el}}$ at the surface (as expected from Figures 4 and 5), but the $\mathrm{Te}_{\mathrm{el}}$ intensity becomes dominant within a sputter time of $5 \mathrm{~s}$ and beyond this the oxide intensity tails off exponentially until no oxide is observed beyond a sputter time of $30 \mathrm{~s}$. This somewhat diffuse oxide/bulk interface can be explained by the sample roughness. 
For both the 0.2 and $2.0 \mathrm{v} / \mathrm{v} \% \mathrm{BM}$ exposures, the passivated surfaces with the shortest BM exposure time $(5 \mathrm{~s})$ exhibit a slightly thinner oxide layer than those exposed for longer times, probably due to there being a slightly thinner Te enriched layer for these surfaces. With regard to determining the oxide thickness, metal oxides are known to generally exhibit much lower sputter rates than metals, so the $\mathrm{TeO}_{2}$ thickness cannot be determined from the profile using the previously determined CZT sputter rate. Hence, oxide thicknesses have been calculated using established procedures based on the BeerLambert expression.

\subsection{Oxide thickness calculation}

Two methods have been used to calculate oxide thickness, a simple Beer-Lambert expression based on photoemission at a single take-off angle and a Beer-Lambert expression based methodology employing a range of take-off angles.

\section{(i) The Beer-Lambert Expression Employing a Single Photoelectron Take-off Angle}

The intensity of photoelectrons emitted from a depth greater than $d$ at an angle $\theta$ to the surface normal is given by the Beer-Lambert expression [15]:

$$
I=I_{0} \exp \left(\frac{-d}{\lambda \cos \theta}\right)
$$

where $I_{0}$ is the intensity from an infinitely thick substrate and $\lambda$ is the photoelectron attenuation length. Manipulation of the Beer-Lambert expression and use of the core level elemental and oxide photoelectron peaks, $I_{e l}$ and $I_{o x}$ respectively, gives rise to the following equation for the oxide thickness, $d_{\mathrm{ox}}$ :

$$
d_{o x}=\lambda \cos \theta \ln \left[1+\left(I_{o x} / I_{e l}\right) /\left(I_{o x}^{\infty} / I_{e l}^{\infty}\right)\right]
$$

where $I_{o x}^{\infty}$ and $I_{e l}^{\infty}$ are the intensities of bulk $\mathrm{TeO}_{2}$ and elemental Te respectively, For calculating overlayer or oxide thicknesses, assuming that $\lambda$ and $\mathrm{I}^{\infty}$ are the same for $\mathrm{Te}$ and $\mathrm{TeO}_{2}$ gives rise to the following simplified expression:

$$
d_{o x}=\lambda_{o x} \cos \theta \ln \left(\frac{I_{o x}}{I_{e l}}+1\right)
$$

The attenuation length of photoelectrons in the oxide layer, $\lambda_{\text {ox }}$, is calculated using Cumpson and Seah's equation given in [16] 


$$
\lambda=0.316 a^{3 / 2}\left[E /\left\{Z^{0.45}(\ln (E / 27)+3)\right\}+4\right]
$$

where $a$ is the lattice parameter $(\mathrm{nm}), E$ is the photoelectron kinetic energy $(\mathrm{eV})$ and $Z$ is the average atomic number of the oxide.

\section{(ii) The Beer-Lambert Expression Employing Multiple Photoelectron Take-off Angles}

With the possibility of acquiring the photoelectron intensities at different take-off angles, then a more rigorous approach can be used to test for the presence of a discrete oxide overlayer and to determine the oxide thickness [15]. The ratio of $I_{o x}^{\infty}$ and $I_{e l}^{\infty}$ can be represent by $R^{\infty}$ which can be found by manipulating some bulk material parameters, given by:

$$
R^{\infty}=\frac{D_{\mathrm{TeO}_{2}} F_{\mathrm{Te}} \lambda_{\mathrm{Te}, \mathrm{TeO} O_{2}}}{D_{\mathrm{Te}} F_{\mathrm{TeO}} \lambda_{\mathrm{Te}, \mathrm{Te}}}
$$

where $D_{\mathrm{Te}}$ and $D_{\mathrm{TeO}_{2}}$ are the elemental Te and $\mathrm{TeO}_{2}$ bulk densities (6.24 and $5.90 \mathrm{~g} / \mathrm{cm}^{3}$ respectively), $F_{T e}$ and $F_{\mathrm{TeO}_{2}}$ are the molar masses of elemental $\mathrm{Te}$ and $\mathrm{TeO}_{2}$ (127.6 and 159.6 respectively) and $\lambda_{\mathrm{Te}, \mathrm{Te}}$ and $\lambda_{\mathrm{Te}, \mathrm{TeO} \mathrm{O}_{2}}$ are the photoelectron attenuation lengths of $\mathrm{Te} 3 \mathrm{~d}_{5 / 2}$ electrons in elemental Te and $\mathrm{TeO}_{2}$, calculated by the Cumpson and Seah equation [16] to be 1.6 and $1.5 \mathrm{~nm}$ respectively. $I_{o x} / I_{e l}$ can be replaced by $\mathrm{R}$ and equation 2 rearranged to give:

$$
\ln \left[1+R / R^{\infty}\right]=\frac{d_{o x}}{\lambda} \frac{1}{\cos \theta}
$$

Then for a set of angle resolved XPS data recorded at different values of take-off angles, $\theta$, the left-hand side of the equation can be plotted against $1 / \cos \theta$. A straight line can be drawn through the data, with the slope corresponding to $d_{o x} / \lambda$. Knowing the attenuation length, the oxide thickness can be determined.

The XPS Thetaprobe angle resolved XPS (ARXPS) capability allows data to be recorded over 16 angles between 25 and $81^{\circ}$ relative to the sample surface normal without rotation of the sample. The slope was determined for take-off angles not exceeding the $50^{\circ}$, to avoid elastic scattering effects. An example of the ARXPS data recorded for a $0.2 \mathrm{v} / \mathrm{v} \% \mathrm{BM}$ exposure for $120 \mathrm{~s}$ seconds followed by a passivation treatment of $15 \mathrm{~s}$ in 30 wt. $\% \mathrm{H}_{2} \mathrm{O}_{2}$ when using equation 6 is given in Figure 8. The plot shows a clear linear relationship and the oxide thickness, $d_{o x}$, calculated from the slope of the straight line was found to be $1.25 \mathrm{~nm}$. 
The $\mathrm{TeO}_{2}$ thickness calculated for the single angle and angle resolved data is shown in Table 1. The oxide thickness trends as a function of exposure time for the different calculation methods are very similar and in both cases the oxide thickness after $5 \mathrm{~s}$ of BM exposure is slightly smaller than other exposure times. The oxide thicknesses calculated from simplified Beer-Lambert expression gives the same oxide thickness for $5 \mathrm{~s}$ of BM exposure for both concentration $(0.2$ and $2.0 \mathrm{v} / \mathrm{v} \%)$. For longer exposure times, the $0.2 \mathrm{v} / \mathrm{v} \% \mathrm{BM}$ exposure exhibits a gradual increase in oxide thickness as the duration time increases. While for $2.0 \mathrm{v} / \mathrm{v} \% \mathrm{BM}$ exposures, beyond $5 \mathrm{~s}$ there is an increase in thickness which remains essentially unchanged for all other exposure times. In both cases $(0.2$ and 2.0 $\mathrm{v} / \mathrm{v} \% \mathrm{BM}$ exposure) the highest value of oxide thickness is around $1 \mathrm{~nm}$.

The oxide thicknesses calculated from ARXPS data are slightly higher than the thickness obtained by simplified Beer-Lambert expression and the trend of increasing oxide thickness for longer BM exposure times is similar to that found for the simplified Beer-Lambert expression. The highest value of oxide thickness for the ARXPS data is $1.25 \mathrm{~nm}$. Considering the methodologies employed, it is likely that the $\ln \left(1+R / R^{\infty}\right)$ vs $1 / \cos \theta$ plot will give more accurate values than the Beer-Lambert expression given in equation 3 as fewer assumptions are made in the former approach. The different values of $D, F$ and and $\lambda$ for $\mathrm{Te}$ and $\mathrm{TeO}_{2}$ are taken into account in Equation 5, whereas the simple Beer-Lambert expression in equation 3 assumes that $\lambda$ and $I^{\infty}$ are the same for $\mathrm{Te}$ and $\mathrm{TeO}_{2}$. Furthermore, the multiple angle approach considers the photoelectron intensity data for a number of take-off angles and the linear regression fit will tend to reduce the experimental error in determination of oxide thickness compared to a single intensity measurement from one take-off angle.

For CZT etched in BM concentrations between 0.2 and $5 \%$, followed by a $30 \mathrm{~s}$ exposure in 30 wt. $\% \mathrm{H}_{2} \mathrm{O}_{2}$, Özsan et al reported oxide thicknesses between 1.6 and $2.8 \mathrm{~nm}$ [8]. Chen et al used a $5 \%$ BM solution followed by $1-15$ minute exposure in $15 \% \mathrm{H}_{2} \mathrm{O}_{2}$ and found oxide thicknesses of between 2.1 and $4.5 \mathrm{~nm}$ [11]. According to Özsan et al, increasing the BM concentration from 2 to 5 $\%$ followed by $30 \mathrm{~s}$ exposure in $30 \mathrm{wt} . \% \mathrm{H}_{2} \mathrm{O}_{2}$ results in only a minimal increase in oxide thickness (from 2.7 to $2.8 \mathrm{~nm}$ ). In this work, using the same simple Beer-Lambert expression employed by the other authors (equation 2), a thickness of $1.0 \mathrm{~nm}$ is reported for exposure in BM concentrations of 0.2 and $2.0 \mathrm{v} / \mathrm{v} \%$ followed by exposure in $30 \mathrm{wt} . \% \mathrm{H}_{2} \mathrm{O}_{2}$ for $15 \mathrm{~s}$. The lower oxide thicknesses reported here compared to those given by the other authors $[8,11]$ could result from the shorter $\mathrm{H}_{2} \mathrm{O}_{2}$ exposure time, but the different attenuation lengths, $\lambda$, are probably the major cause of discrepancy. Özsan et al [8] used the simpler, empirical Seah and Dench [17] approach, which yields a value of $2.6 \mathrm{~nm}$ for $\lambda$, rather than the more refined Cumpson and Seah expression [16] employed in this work, which gives a value of $1.5 \mathrm{~nm}$. 


\section{Conclusions}

CZT single crystals have been exposed to etching treatments using a BM concentration of 0.2 and $2.0 \mathrm{v} / \mathrm{v} \%$ for exposure times between 5 and $120 \mathrm{~s}$. XPS depth profiles have shown that both treatments resulted in the formation of a Te rich surface region. The enriched Te layer thickness was accurately determined by calibrating the CZT sputter rate against a CdTe layer of known thickness. Varying the exposure time had no effect on the thickness of the Te rich region, which was found to extend to a depth of $1.3 \pm 0.2 \mathrm{~nm}$ for the $0.2 \mathrm{v} / \mathrm{v} \% \mathrm{BM}$ etch and $1.8 \pm 0.2 \mathrm{~nm}$ for the $2.0 \mathrm{v} / \mathrm{v} \% \mathrm{BM}$ etch. The invariant effect of exposure time between 5 and $120 \mathrm{~s}$ is considered to be due to the rapid etching process of such a thin Te enriched layer. The similarity in the surface compositions for the two different $\mathrm{BM}$ concentrations is ascribed to the comparable $\mathrm{pH}(\mathrm{pH} \approx 3)$ of both solutions but the increase in the Te rich layer thickness for the higher concentration of BM is probably due to this solution having a slightly lower $\mathrm{pH}$.

The $\mathrm{BM}$ exposed surfaces were passivated using a $15 \mathrm{~s} \mathrm{H}_{2} \mathrm{O}_{2}$ treatment. A thin $\mathrm{TeO}_{2}$ layer was formed. XPS depth profiles showed there to be little change in the oxide thickness between the surfaces BM etched at different concentrations or etch times, but the $5 \mathrm{~s}$ exposure in both $0.2 \%$ and 2 $\mathrm{v} / \mathrm{v} \% \mathrm{BM}$ gave rise to a slightly thinner oxide layer than the longer BM exposure times. Determination of the oxide thickness using a Beer-Lambert expression and single photoelectron takeoff angle showed a similar trend to that using Beer-Lambert expression and multiple photoelectron take-off angles. For $\mathrm{H}_{2} \mathrm{O}_{2}$ treated samples, the former method gave an oxide thickness in the range of $1.0 \pm 0.07 \mathrm{~nm}$ whilst the latter yielded an oxide thickness of $1.25 \pm 0.12 \mathrm{~nm}$.

The results presented in this paper give accurate values for the thickness of both the enriched Te layer (following BM exposure at different concentrations) and the $\mathrm{H}_{2} \mathrm{O}_{2}$ generated oxide layer. Such data is important to workers in the field of device fabrication where a balance between low leakage current and good charge collection is required from this surface region. Hence, this study is of importance for further research work on the determination of optimum oxide thickness and composition for improved device performance. 


\section{Acknowledgements}

The authors would like to thank Professor Stuart Irvine's group at the Centre for Solar Energy Research, Glyndŵr University, UK, for providing the MOCVD CdTe layer for XPS calibration purposes and Dr John Wolstenholme from Thermo Scientific for useful discussions regarding oxide thickness calculations. 


\section{References}

[1] Y. Eisen, A. Shor, J. Cryst. Growth, 184 (1988) 1302.

[2] T. E. Schlesinger et al, Mat. Sci. Eng., 32 (2001) 103.

[3] P. J. Sellin, Nuc. Inst. Methods Phy. Res., 513 (2003) 322.

[4] H. Prettyman et al, SPIE Proceedings, Vol., 4507 (2001) 22.

[5] G. Tepper et al, Proc. SPIE, 4507 (2001) 79.

[6] H. Chen et al, J. Vac. Sci. Technol., A 17 (1999) 97.

[7] A. Etcheberry et al, J. Cryst. Growth, 184-185, (1998) 213.

[8] M. E. Özsan et al, Surf. Inter. Anal., 42 (2010) 795.

[9] A .A. Rouse et al, IEEE Trans. Nucl. Sci., 49, (2002) 2005.

[10] H. Bensalah et al, Appl. Surf. Sci., 257 (2011) 4633.

[11] K. T. Chen et al, J. Vac. Sci. Tech., A 15 (1997) 850.

[12] C. K. Egan et al, J. Appl. Phy., 108 (2010) 024310.

[13] R.A. Berrigan et al, J. Cryst. Growth, 195 (1998) 718.

[14] A. Bensouici et al, J. Cryst. Growth, 312 (2010) 2098.

[15] J.Watts and J.Wolstenholme, An Introduction to Surface analysis by XPS and AES, Wiley, (2003) p.83

[16] P. J. Cumpson and M. P. Seah, Surf. Inter. Anal., 25 (1997) 430.

[17] M. P. Seah, W. A. Dench, Surf. Inter. Anal.1 (1979) 2. 


\begin{tabular}{|c|c|c|c|c|c|c|c|}
\hline \multirow[b]{3}{*}{$\begin{array}{l}\text { Single angle } \\
\text { (simplified Beer- } \\
\text { Lambert expression) }\end{array}$} & \multirow[b]{2}{*}{$\underbrace{\text { BM exposure }}_{\substack{\text { duration } \\
\text { concentration }}}$} & \multicolumn{6}{|c|}{$\begin{array}{c}\text { Average oxide thickness }(\mathrm{nm}) \\
( \pm 0.07 \mathrm{~nm} \text { for single angle and } \pm 0.12 \mathrm{~nm} \text { for } \\
\text { ARXPS })\end{array}$} \\
\hline & & $5 \mathrm{~s}$ & $15 \mathrm{~s}$ & & $60 \mathrm{~s}$ & 12 & \\
\hline & $\begin{array}{l}0.2 \% \\
2 \%\end{array}$ & $\begin{array}{l}0.83 \\
0.84\end{array}$ & $\begin{array}{l}0.93 \\
.99\end{array}$ & $\begin{array}{l}0.96 \\
0.98\end{array}$ & & $\begin{array}{l}0.97 \\
0.99\end{array}$ & $\begin{array}{l}1.05 \\
1.00\end{array}$ \\
\hline Angle resolved XPS & $\begin{array}{l}0.2 \% \\
2 \%\end{array}$ & $\begin{array}{l}0.89 \\
1.04\end{array}$ & $\begin{array}{l}1.03 \\
1.17\end{array}$ & $\begin{array}{l}1.09 \\
1.23\end{array}$ & & $\begin{array}{l}1.13 \\
1.25\end{array}$ & $\begin{array}{l}1.25 \\
1.25\end{array}$ \\
\hline
\end{tabular}

Table 1: Comparison of the $\mathrm{TeO}_{2}$ thickness calculated by a simplified Beer-Lambert expression (single take-off angle) compared to ARXPS data (range of take-off angles) after exposing CZT in $0.2 v / v \%$ and $2 v / v \%$ BM for varying times followed by a 15 s passivation treatment in $30 \mathrm{wt} \% \mathrm{H}_{2} \mathrm{O}_{2}$. 


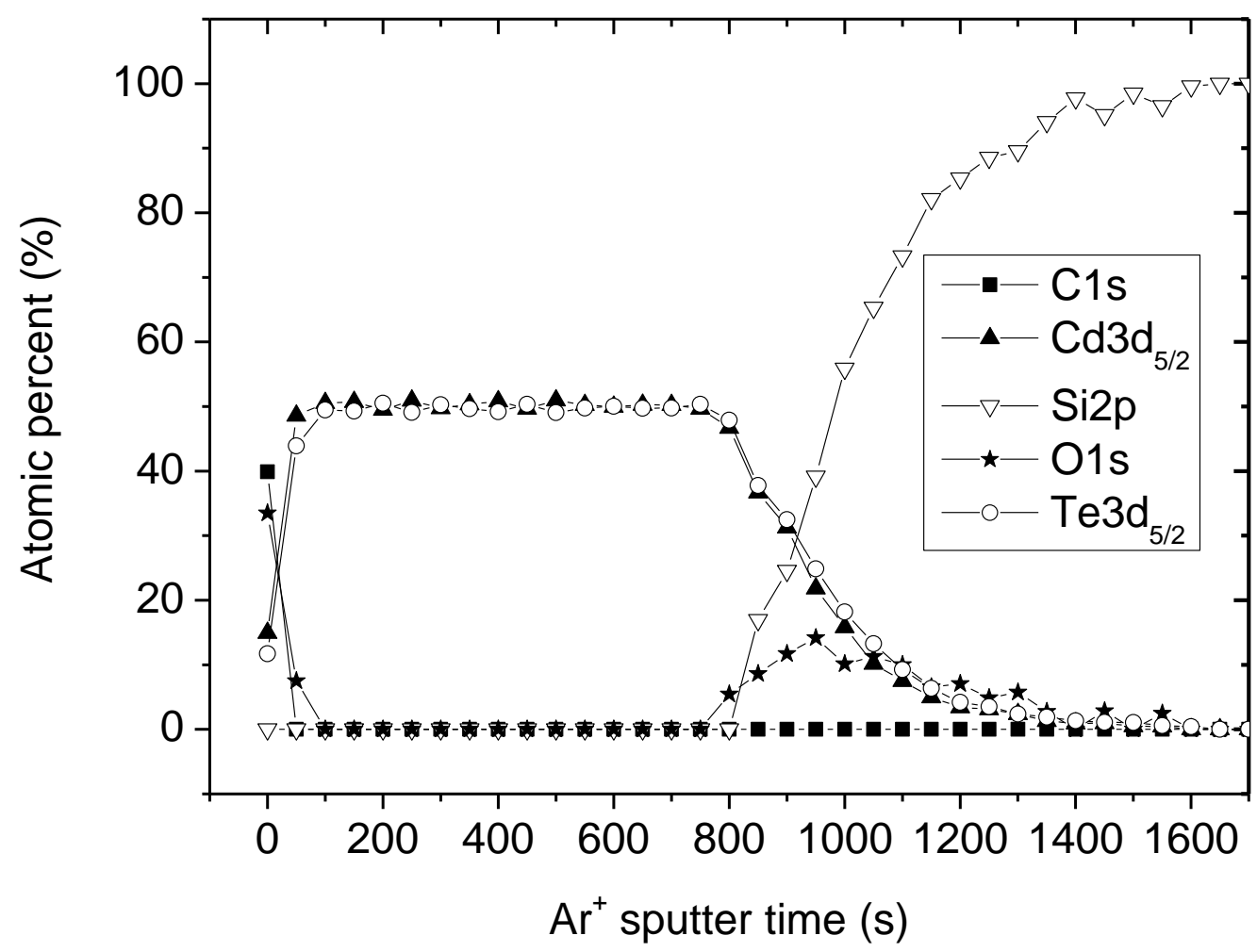

Figure 1.XPS depth profile of an MOCVD deposited CdTe layer of $135 \mathrm{~nm}$ thickness. The CdTe etch rate of $3 \mathrm{kV} \mathrm{Ar}^{+}$ions at a beam current density of $11.1 \mu \mathrm{A} / \mathrm{cm}^{2}$ was determined to be $0.15 \mathrm{~nm} / \mathrm{s}$. 


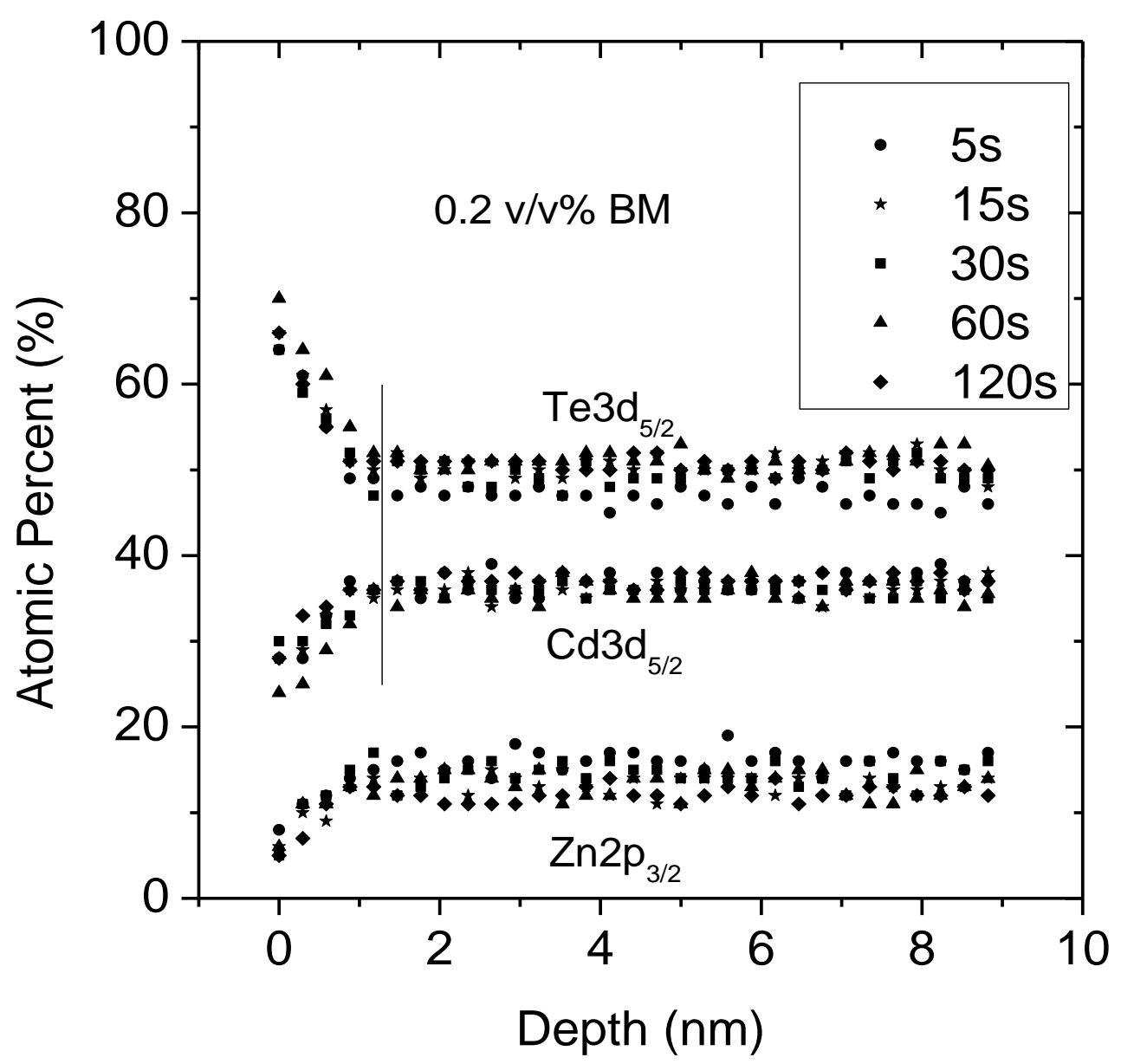

Figure 2. XPS depth profiles of CZT exposed to $0.2 v / v \%$ BM for various time periods $(5 \mathrm{~s}, 15 \mathrm{~s}$, $30 \mathrm{~s}, 60 \mathrm{~s}$ and $120 \mathrm{~s})$. 


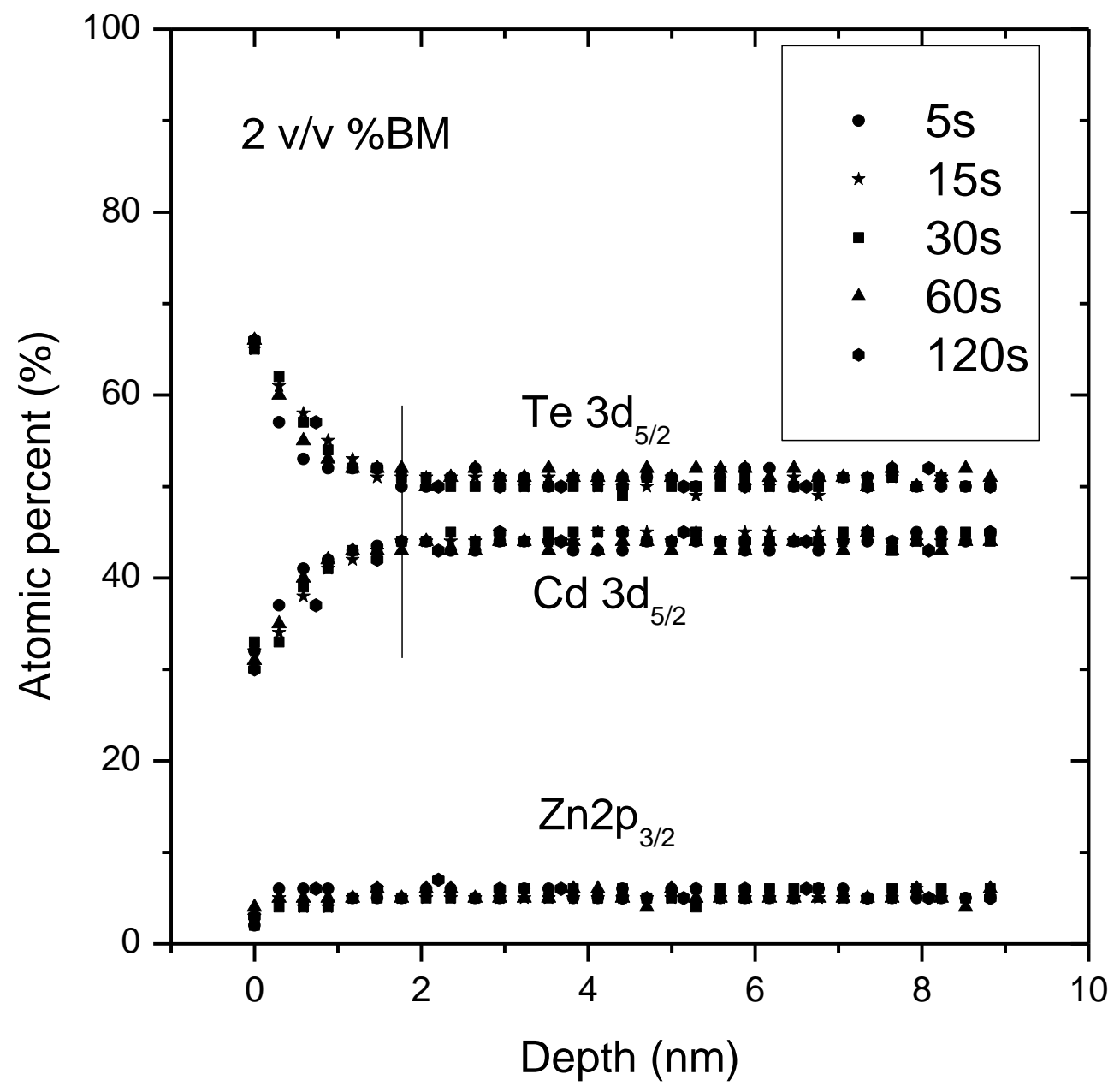

Figure 3. XPS depth profiles of CZT exposed to 2 v/v\% BM for various exposure periods ( 5 , $15 s, 30 s, 60 s$ and $120 s$ ). 


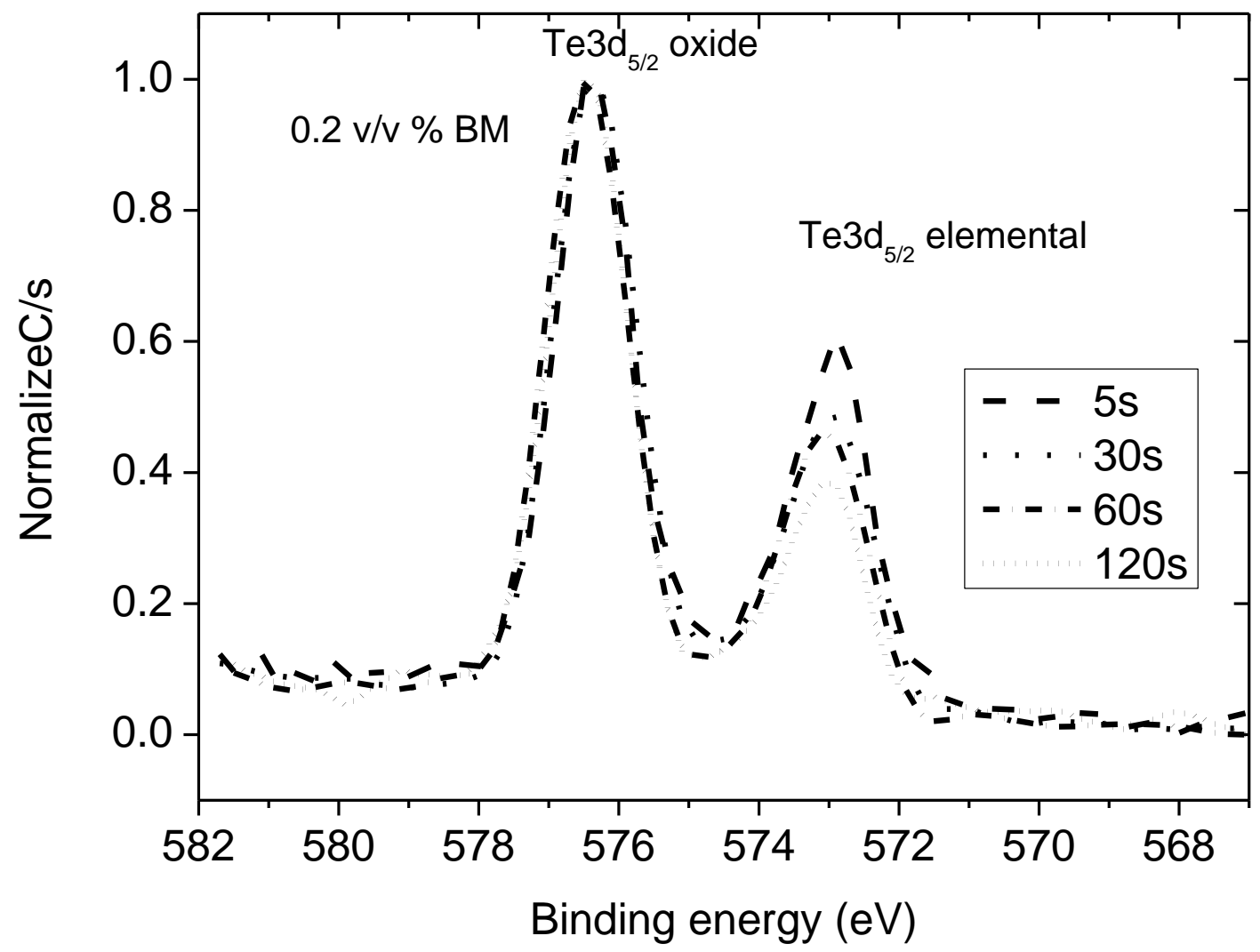

Figure 4. XPS Te $3 d_{5 / 2}$ peak for CZT exposed in $0.2 v / v \%$ BM for different times (5-120s) followed by 15 seconds exposure in $30 \mathrm{wt} \% \mathrm{H}_{2} \mathrm{O}_{2}$ procedures based on the Beer-Lambert expression. 


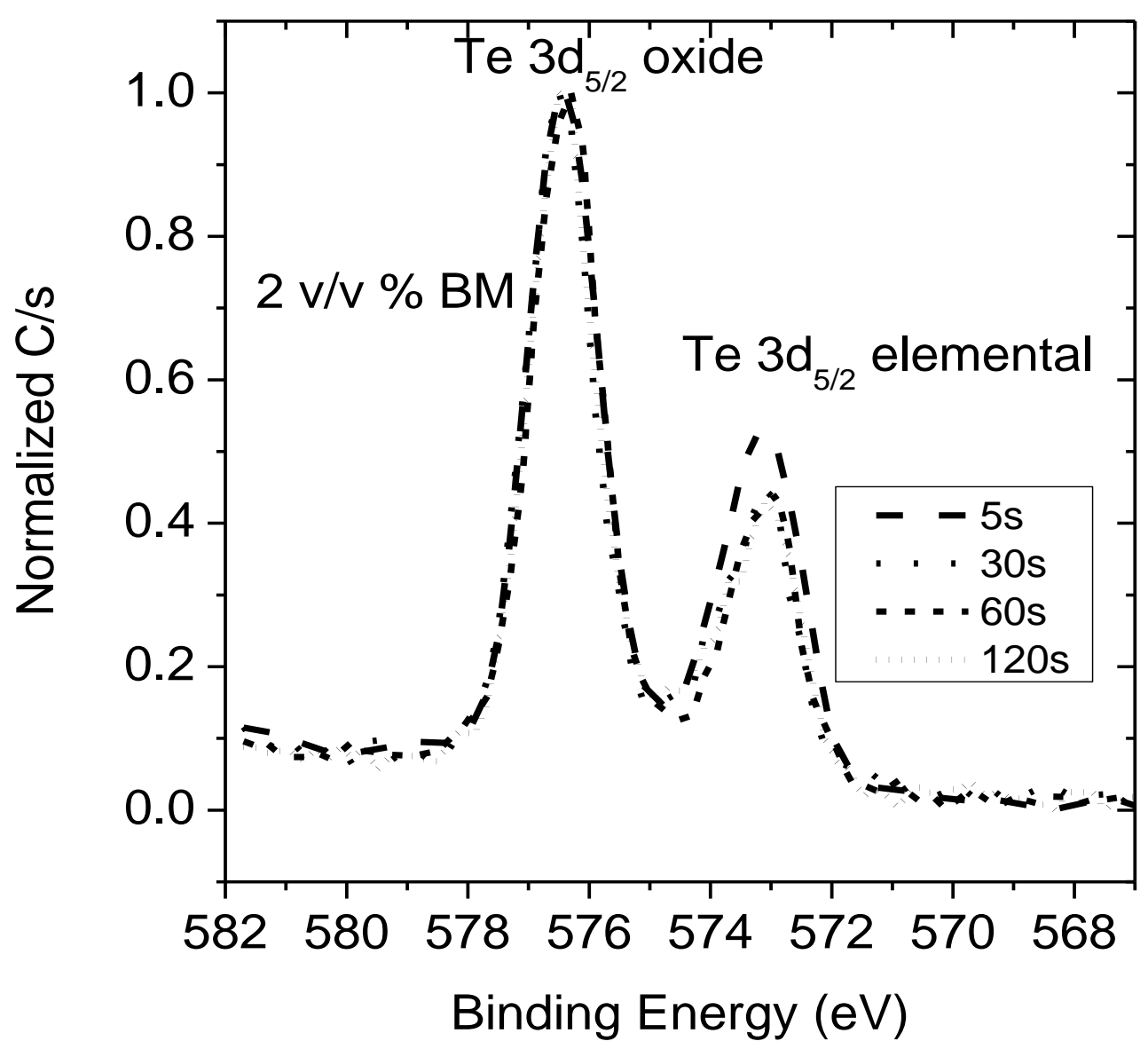

Figure 5. XPS Te $3 d_{5 / 2}$ peak for CZT exposed in $2.0 v / v \%$ BM for different times (5-120s) followed by 15 seconds exposure in $30 \mathrm{wt} \% \mathrm{H}_{2} \mathrm{O}_{2}$. 


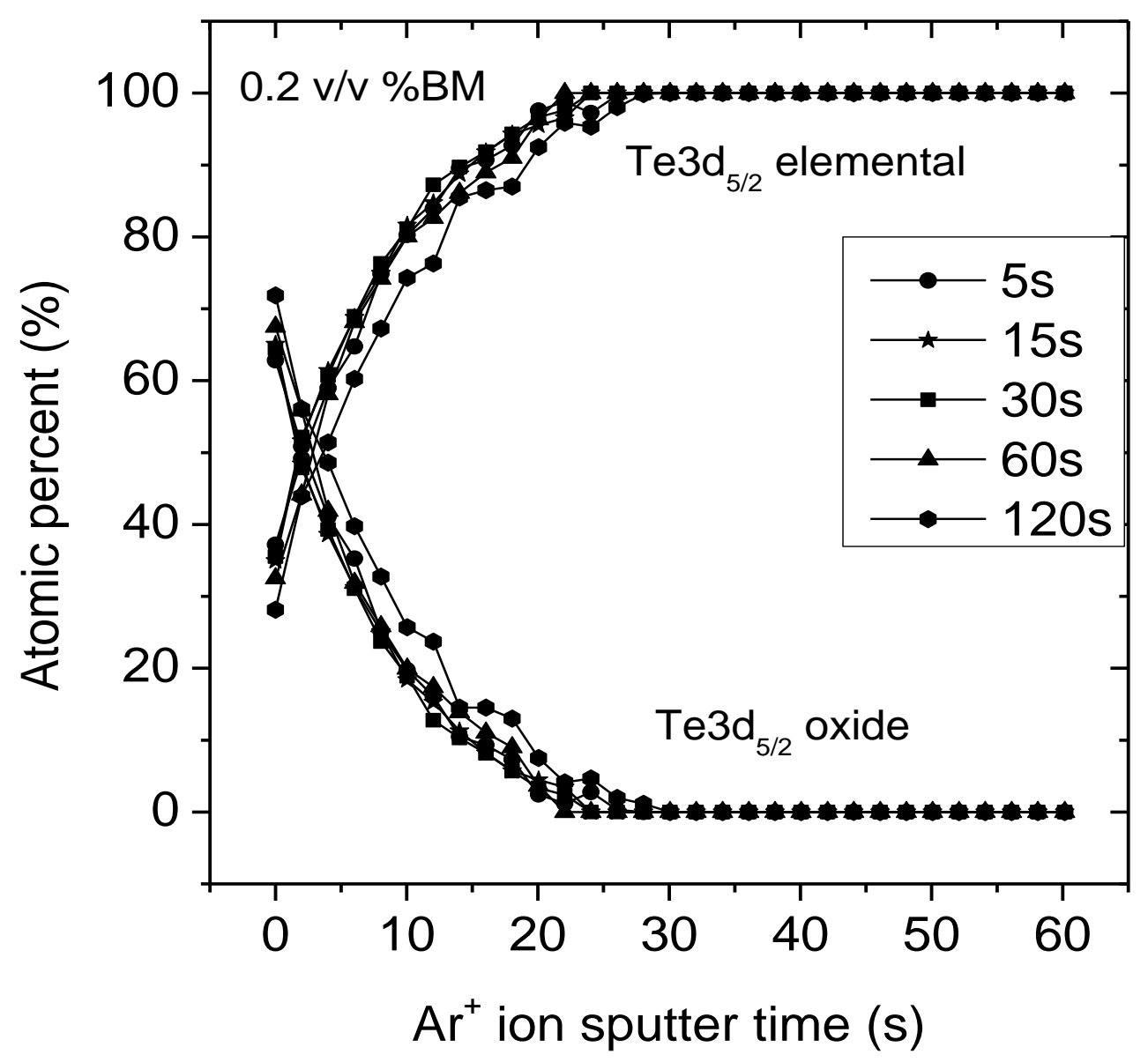

Figure 6: XPS depth profile (using CZT Te 5/2 $_{2}$ bulk and oxide peaks) of CZT surfaces exposed to $0.2 \mathrm{v} / \mathrm{v} \% \mathrm{BM}$ for various times followed by a $15 \mathrm{~s}$ passivation treatment in $30 \mathrm{wt} \% \mathrm{H}_{2} \mathrm{O}_{2}$. 


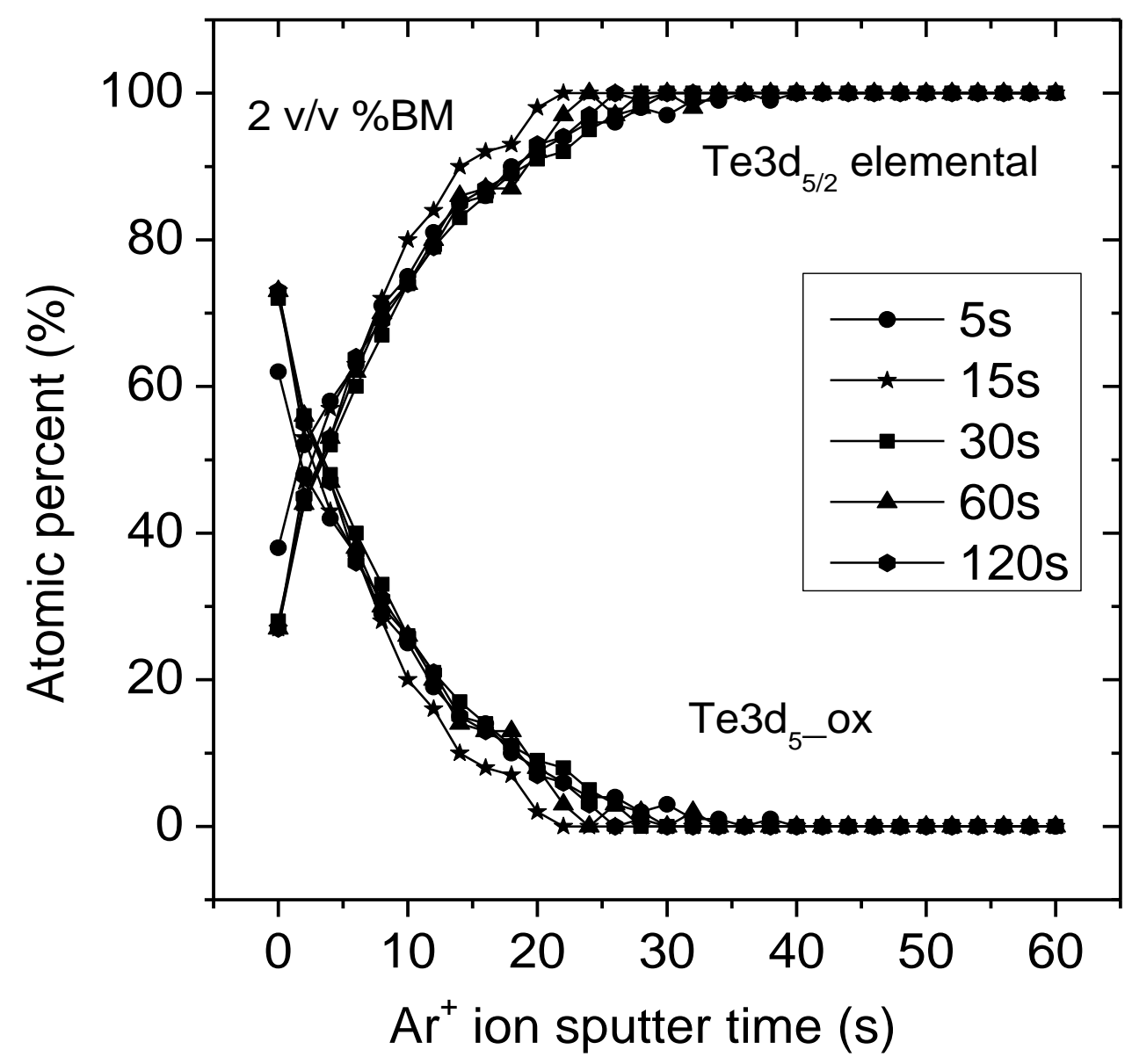

Figure 7: XPS depth profile (using CZT Te ${ }_{5 / 2}$ bulk and oxide peaks) of CZT surfaces exposed to $2.0 \mathrm{v} / \mathrm{v} \% \mathrm{BM}$ for various times followed by a $15 \mathrm{~s}$ passivation treatment in $30 \mathrm{wt} \% \mathrm{H}_{2} \mathrm{O}_{2}$. 


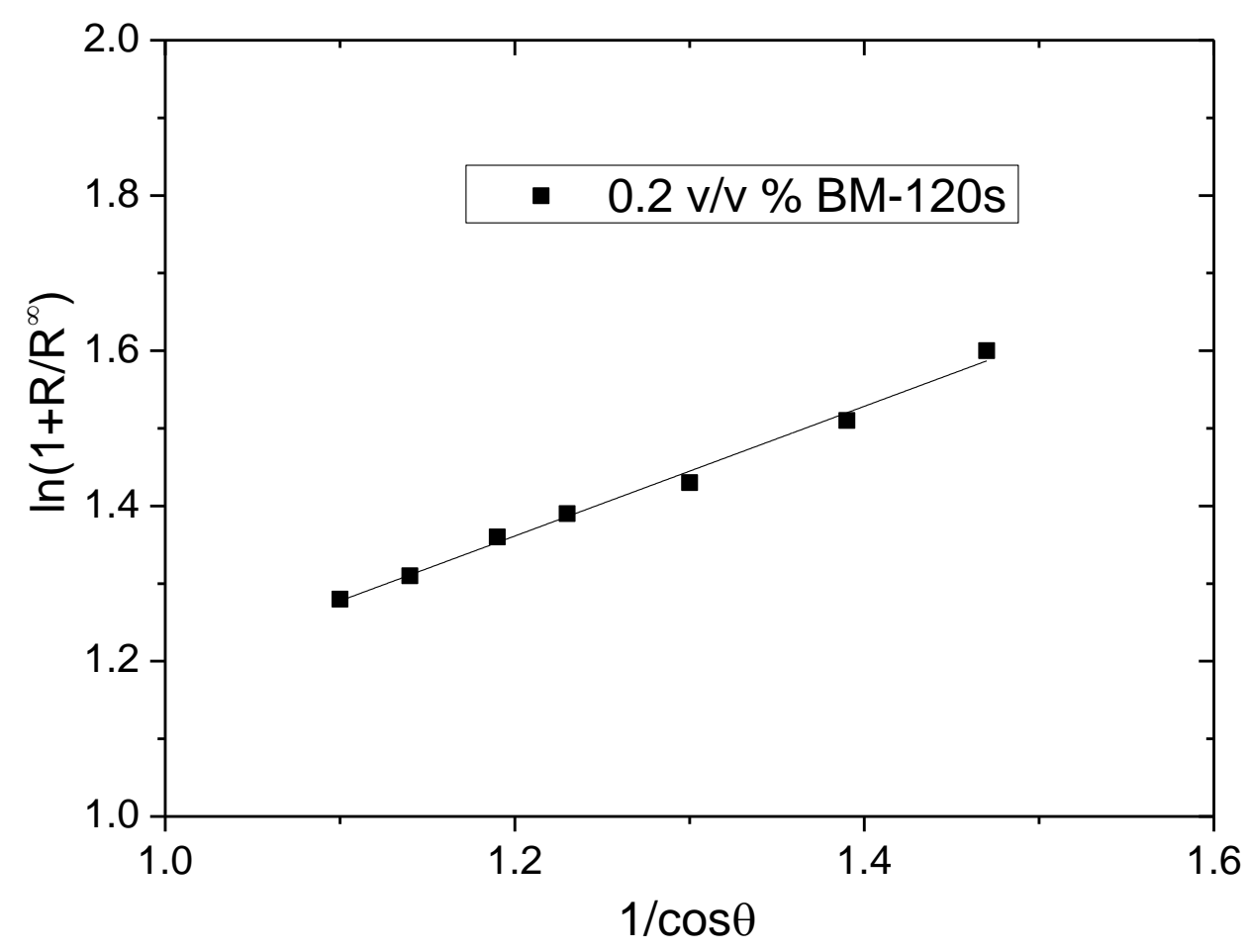

Figure 8: ARXPS plot to determine the oxide thickness, $d_{o x}$, of a CZT surface exposed in 0.2 v/v\% BM for 120s seconds followed by passivation in 30 wt.\% $\mathrm{H}_{2} \mathrm{O}_{2}$ for 15 seconds. 